\title{
Erratum: Endoscopic Endonasal Transplanum- Transtuberculum Sellae Approach for the Resection of a Diaphragma Sellae Meningioma
}

\author{
Juan M. Revuelta Barbero ${ }^{1}$ Alaa S. Montaser ${ }^{1,2} \quad$ Alexandre B. Todeschini ${ }^{1}$ Mostafa Shahein ${ }^{1}$
} Bradley A. Otto ${ }^{1,3}$ Ricardo L. Carrau ${ }^{1,3}$ Daniel M. Prevedello ${ }^{1,3}$

${ }^{1}$ Department of Neurological Surgery, Wexner Medical Center, The Ohio State University, Columbus, Ohio, United States

${ }^{2}$ Department of Neurosurgery, Ain Shams University Faculty of Medicine, Cairo, Egypt

${ }^{3}$ Department of Otolaryngology-Head and Neck Surgery, Wexner Medical

Center at The Ohio State University, Columbus, Ohio, United States

J Neurol Surg B 2018;79:e2.
Address for correspondence Daniel M. Prevedello, MD, Department of Neurological Surgery, Wexner Medical Center at The Ohio State University, N-1049 Doan Hall, 410 West 10th Avenue, Columbus, OH 43210, United States (e-mail: daniel.prevedello@osumc.edu).
ERRATUM
It has been brought to our attention that the last name of Juan M. Revuelta Barbero was identified incorrectly in the above article published online in Volume 79, Issue S3 of the Journal of Neurological Surgery Part B (DOI: 10.1055/s-0038-1624587). The first name should be "Juan", middle name "M.", and last name should be "Revuelta Barbero". 